\title{
The Use of Supratrochlear Artery Based Myofascial Forehead Flap for Treatment of a Rhinophyma Case
}

\section{Umut Tuncel*, Murat Gümüs and Alper Kurt}

Department of Plastic Reconstructive and Aesthetic Surgery, Samsun Education and Research Hospital Samsun/Turkiye

\section{Abstract}

Rhinophyma is a disease going with subcutaneous tissue thickening of the nose, including sebaceous gland hypertrophy and fibrosis. Progressive enlargement and irregular thickening of the nasal skin is characteristic of the disease. Surgical excision is commonly used treatment method. In the present case, nasal dorsal and alar defect after treated with totally excision and full-thickness skin graft of rhinophyma mass were repaired by using supratrochlear artery based myofascial forehead flap with a little skin island.

\section{Publication History:}

Received: December 26, 2016

Accepted: September 20, 2017

Published: September 22, 2017

\section{Keywords:}

Rhinophyma, Forehead flap, Supratrochlear artery

\section{Introduction}

Rhinophyma is an extremely deformating condition of the nose and considered to be the most severe expression of the final stage of acne rosacea, which is characterized by a progressive painless hyperplasia of the sebaceous glands and connective tissue of the nose [1]. Although the etiology of rhinophyma is unknown, it is commonly believed to be a severe end-stage of a skin condition known as rosacea [2,3].

The supratrochlear artery based forehead flap has become commonly preferred reconstructive option when dealing with repairing large nasal defects [4-6]. Knowledge of the anatomical course of the supratrochlear artery allows for more precise flap design, greater mobility, and increased pedicle length [7].

We present a case treated with supratrochlear artery based myofascial forehead flap along with full-thickness skin graft and recommend this technique as a useful, safe, and efficient method for the treatment of severe and moderate rhinophyma.

\section{Patient and Method}

A 50-year-old man presented with a 10-year history of progressive bulbous overgrowth of the nose. He had a history of cystic acne as a teenager. The man had never undergone any laser or surgical interventions and the nose kept slowly enlarging. No other medical problem was reported, and there was no family history of rosacea. Nodulocystic and scarred areas, also large dilated pores on the cheeks were observed on the physical examination. The nose was composed of an exophytic bulbous mass with obliteration of the normal nose contour (Figure 1). The rhinophyma mass was completely excised and the defect was repaired by a full-thickness skin graft. However, skin graft on the nasal dorsum was showed to be necrosis after the surgery (Figure 2). A supratrochlear artery based myofascial forehead flap along with full-thickness skin graft was planned to close the defect. Also, a skin island at tip of the myofascial flap was planned for the reconstruction of nasal alar defect.

The procedure was performed under general anesthesia. The wound was debrided and a subcutaneous paramedian forehead flap was designed according to the size of defect area on the nasal dorsum and nasal ala. Before the flap was raised, supratrochlear artery was dissected on the location about $2 \mathrm{~cm}$ lateral to the midline near the medial eyebrow and the flap pedicle of approximately $1 \mathrm{~cm}$ width was



"Corresponding Author: Dr. Umut Tuncel, Department of Plastic Reconstructive and Aesthetic Surgery, Samsun Education and Research Hospital Samsun/ Turkiye, Tel: + 90532724 9384; E-mail: drumuttuncel@gmail.com

Citation: Tuncel U, Gümüs M, Kurt A (2017) The Use of Supratrochlear Artery Based Myofascial Forehead Flap for Treatment of a Rhinophyma Case. Int J Surg Surgical Porced 2: 121. doi: https://doi.org/10.15344/2456-4443/2017/121

Copyright: @ 2017 Tuncel. This is an open-access article distributed under the terms of the Creative Commons Attribution License, which permits unrestricted use, distribution, and reproduction in any medium, provided the original author and source are credited. 
prepared, including frontalis muscle cuff. Then, subcutaneous dissection was made above the flap pedicle toward the orbital rim and to $4 \mathrm{~cm}$ above the first incision where the pedicle was dissected. The frontal muscle was not involved in remaining of flap area except around the pedicle. A $1 \times 1 \mathrm{~cm}$ island of skin was also involved to top of the flap for reconstruction of nasal alar defect. After the flap dissection was completed, subcutaneous myofascial forehead flap was raised and the flap was transposed to the nasal dorsal and alar defect (Figure 3). After the nasal alar defect was repaired by island of skin, a fullthickness skin graft was harvested from the left supraclavicular area and placed on the myofascial flap for repairing the nasal dorsal defect. The donor sites were closed primarily and the wound was followed using an oily dressing. The postoperative period was uneventful, flap and skin graft was totally survived with an acceptable aesthetic outcome. At a 3 month-follow-up period, a scar was present on the nasal dorsum, but the overall nasal contour was well-restored.

\section{Discussion}

A wide range of surgical approaches to rhinophyma has been described including cryosurgery, electrosurgery, resection with heated knives and loops, dermabrasion, scalpel and razor blade excision, $\mathrm{CO}_{2}$ laser surgery, and argon laser surgery [1-3]. End results and morbidity are similar with various techniques. Because of this, the commonly used treatment of the disease is surgical excision and skin graft in plastic surgical practice.

The primary aim for the treatment of the nasal defects is to provide an aesthetically acceptable result causing minimal donor site morbidity [8]. Split of full-thickness skin grafts or a various type of local flaps can be used for the medium or large size defects of nose. The reconstruction of choice on nasal dorsal defects is generally local flaps or forehead flap. Nasolabial flaps are usually preferred in the defects of the nasal ala, tip or sidewall among the full-thickness nasal defects. The application of this flap as a classic folded flap with or without cartilage graft support causes bulky formation because the distal aspect of the flap must be folded. Another treatment alternative in the repair of large full-thickness nasal defects is the free flaps. However, because the tissue transplanted from a distant region as a free flap has disadvantages such as color incompatibility, long duration of surgery, and the need for special equipment, they are obviously more suitable for cases in a reconstruction with local-regional flaps is not an option [8].

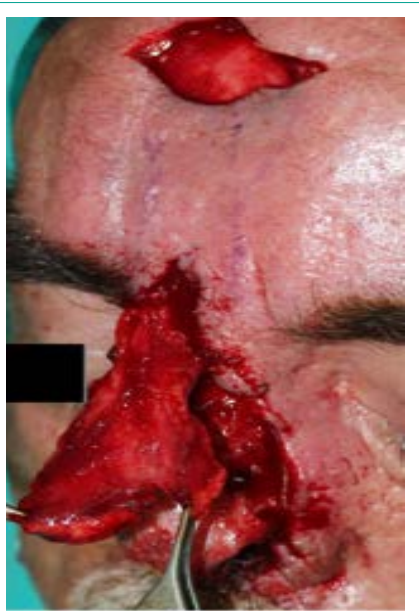

Figure 3: Supratrochlear artery based myofascial forehead flap was raised and transposed to the defect.
The forehead flap and its various modifications have widely been used to cover the nasal defect. The anatomic studies demonstrated the position and course of the supratrochlear artery, running 1.7 to 2.2 $\mathrm{cm}$ lateral to the midline in a vertical vector [9]. The artery runs in the submuscular plane to a more superficial, subcutaneous position beginning $1 \mathrm{~cm}$ above the brow. Knowledge of the anatomical course of the supratrochlear artery allows for more precise flap design, greater mobility, and creased pedicle length [5]. The paramedian forehead flap is the best of choice or reconstructing of defect greater than $2 \mathrm{~cm}$. The skin defect size in our patient was $2^{\star} 2 \mathrm{~cm}$ and there was full-thickness defect involved perichondrium and periost. Thus, we planned to perform a supratrochlear artery based forehead flap to cover this defect. However we preferred a myofascial design flap so that minimal donor site morbidity such as visible scar and frontal deformity due to frontalis muscle deficiency. In traditional flap design, the flap can't be optimal when an optimal cosmetic result is required, because only a less-than-optimal cosmetic outcome can be achieved in one stage [10]. Another alternative can be expanded flap design but it requires two-stage procedure and the relatively long treatment period can't be comfortable for patient. Only two skin incisions were used to raise the flap, and the frontalis muscle was involved the flap only around the pedicle. In addition, alar defect was also reconstructed owing to little skin-island on top of the myofascial flap. The present design of the flap previously presented by Ebrahimi et al. [11]. In that similar study, the authors included periosteum in pedicle for better vascularity. However we did not used periosteum while flap pedicle was raised because we considered myofascial pedicle with supratrochlear artery is enough to vascularity of the flap. In addition, we think that this pedicle can get more ease rotation of the flap in especially glabellar region where the flap pedicle will be two folded in. Venous congestion was observed in early postoperative period but it was resolved in 3 days. The skin-island is not able to add to the flap as it isn't needed. Otherwise, the flap pedicle did not skeletonize in the present method and the flap was created large enough to the defect while raising the flap, so venous drainage was uneventful for postoperative period. All donor sites were closed primarily. Thus, a satisfactory and acceptable aesthetic result was obtained postoperatively.

In conclusion, this modified method is less invasive and simpler compared to the original procedure. The supratrochlear artery based myofascial flap was found to be safely and reliable reconstructive method for the treatment of full-thickness dorsal nasal defect when the defect size is moderate and large or other methods are not available.

\section{Competing Interests}

The authors declare that they have no competing interests.

\section{References}

1. Sadick H, Goepel B, Bersch C, Goessler U, Hoermann K, et al. (2008) Rhinophyma: diagnosis and treatment options for a disfiguring tumor of the nose. Ann Plast Surg 61: 114-120.

2. Shah A, Lakhani R, Panesar J (2013) Rhinophyma-a patient case study. J Vis Commun Med 36: 128-131.

3. Husein-El Ahmed H, Armijo-Lozano R (2013) Management of severe rhinophyma with sculpting surgical decortication. Aesthetic Plast Surg 37: 572-575.

4. Pawar SS, Kim MM (2013) Updates in forehead flap reconstructions of facial defects. Curr Opin Otolaryngol Head Neck Surg 21: 384-388. 
Citation: Tuncel U, Gümüs M, Kurt A (2017) The Use of Supratrochlear Artery Based Myofascial Forehead Flap for Treatment of a Rhinophyma Case. Int J Surg Surgical Porced 2: 121. doi: https://doi.org/10.15344/2456-4443/2017/121

Page 3 of 3

5. Jellinek NJ, Nguyen TH, Albertini JG (2014) Paramedian forehead flap: advances, procedural nuances, and variations in technique. Dermatol Surg 40: S30-S42.

6. Cook JL (2014) The reconstruction of the nasal ala with interpolated flaps from the cheek and forehead: design and execution modifications to improve surgical outcomes. Br J Dermatol 2: 29-36.

7. Correa BJ, Weathers WM, Wolfswinkel EM, Thornton JF (2013) The forehead flap: the gold standard of nasal soft tissue reconstruction. Semin Plast Surg 27: 96-103.

8. Selçuk CT, Durgun M, Özalp B, Bozkurt M (2013) Cartilage-supported paramedian forehead flaps for reconstruction of full-thickness nasal defects. J Craniofac Surg 24: 425-427.

9. Yu D, Weng R, Wang H, Mu X, Li Q (2010) Anatomical study of forehead flap with its pedicle based on cutaneous branch of supratrochlear artery and its application in nasal reconstruction. Ann Plast Surg 65: 183-187.

10. Menick FJ (2002) A 10-year experience in nasal reconstruction with the three-stage forehead flap. Plast Reconstr Surg 109: 1839-1855.

11. Ebrahimi A, Kalantar Motamedi MH, Nejadsarvari N, Shams Koushki E (2012) Subcutaneous forehead island flap for nasal reconstruction. Iran Red Crescent Med J 14: 271-275.

This article was originally published in a special issue:

Plastic Surgery

Handled by Editor(s):

Prof. SuRak Eo

Department of Plastic \& Reconstructive Surgery

DongGuk University Medical Center

South Korea 